\title{
蛋白质二硫键异构酶的二硫键不是其 活力所必需*
}

\author{
余宣传 \\ (中国科学院生物物理研究所, 北京 100101)
}

\section{关键词核桾核酸醇、必需䘪基、二硫键、氧化还原缓冲液、蛋白质二硫键异构醉}

蛋白质二硫键异构酶 (Protein disulfide isomerase, PDI, EC 5.3.4.1) 催化蛋白质分子中 巯基与二硫键的交换反应, 并被普遍认为催化细胞内蛋白质生物合成过程中天然二硫键的形 成. 虽然根据 PDI 的 cDNA 序列所推断的氨基酸序列中, 每个亚基只含 6 个 Cys 残基, 完全 还原的 PDI 在不同的实验室所测得的流基数目却都为 $7^{[1-3]}$, Carmichael ${ }^{[2]}$ 等报道, 不经还 原剂还原的 PDI 含有三个自由巯基和两对二硫键, 但 Gilbert 等报道 PDI 含有三对二硫键 ${ }^{[3]}$. PDI 亚基中含有两段高度重复序列 1- 100 和 350-450, 这两段序列和大肠杆菌的硫氧还蛋 白的相应序列十分相似, 其中 -WCGHCK- 与硫氧还蛋白活性中心 -WCGPCK- 极为类似 ${ }^{[1]}$. Hawkins 等 ${ }^{[4]}$ 报道, 在无变性剂存在时, PDI 经 DTT 还原后, 可被 IAA (Iodoacetic acid) 修 饰的疏基只有这 2 个片段中的 Cys-32 和 Cys-379, 这两个䘪基是 PDI 活性所必需的, 并且是 相互独立的. 他们认为这 2 个片段是 PDI 的活性中心. 用定点突变方法, 将 Cys 用 Ser 代 替, 也表明两个活性中心是独立起作用的 ${ }^{[5]}$. 按 Hawkins 和 Freedman ${ }^{[6]}$ 的方法提纯的 PDI, 这两个片段中的 Cys 以二硫键形式存在 ${ }^{[3,4]}$, 二硫键是否是 PDI 活力所必需的, 目前还没有报 道. 用 GSH/GSSG 比例组成不同的氧化还原缓冲液可以控制 PDI 在反应过程中的氧化还原 程度, 从而研究 PDI 分子中疏基二硫键状态与其活力的关系. Lyles 和 Gilbert ${ }^{[7]}$ 曾报道, 氧化 还原缓冲液中, GSH 与 GSSG 的比例为 1:0.2 时, PDI 活力最大. Hawkins 等 ${ }^{[8]}$ 也曾报道类 似的结果, 但他们都没有测定相应条件下 PDI 分子本身所具有的毓基数目. 本文测定了氧化 还原缓冲液对 PDI 活性和疏基的影响, 结果表明, PDI 的二硫键不是其异构酶活力所必需 的, 还原型的 PDI 活力最高.

\section{1 材 料与 方 法}

核糖核酸酶 (Ribonuclease, RNase), 还原型和氧化型谷胱甘肽 (GSH, GSSG)、盐酸胍 $(\mathrm{GuHCl})$ 为 Sigma 公司产品. 二疏基苏糖醇 (dithiothreitol, DTT)、Serva、碘乙酰胺菜酚 (4Iodo-acetamido-1-naphthol, IAN) 为 Polyscience 公司产品, DTNB (5, 5' -dithiobis (2-nitrobenzoic acid)), Merck 等为国产分析纯. PDI 是按照 Lambert 和 Freedman ${ }^{[6]}$ 的方法从新鲜牛

1993-04-16 收稿, 1993-10-20 收修改稿.

*国家自然科学基金资助项目. 
肝中提取, 用二硫键错接核糖核酸酶 (sR Nase) 作底物, 比活为 900 单位 $/ \mathrm{g}$.

\section{1 放基的测定}

PDI 用 $1 \mathrm{mmol} / \mathrm{L}$ DTT 在 $\mathrm{pH} 8.0$ 含 $1 \mathrm{mmol} / \mathrm{L} \mathrm{EDTA}$ 的 $0.1 \mathrm{~mol} / \mathrm{L}$ Tris $-\mathrm{HCl}$ 的缓冲液中于 $37^{\circ} \mathrm{C}$ 还原 $30 \mathrm{~min}$ 后, 用 Sephadex G25 离心凝胶过滤除去过量的 DTT, 得到完全还原的 PDI. PDI 用含 $2 \mathrm{mmol} / \mathrm{L} \mathrm{EDTA}$ 和不同 GSH/GSSG 比例的 $\mathrm{pH} 7.5$ 的 $0.2 \mathrm{~mol} / \mathrm{L}$ 磷酸钠缓冲液处理 $10 \mathrm{~min}$ 后, 用 Sephadex G25 离心凝胶过滤除去过量的还原剂, 得到不同氧化还原程度的 PDI. 在 $\mathrm{pH} 8.0$ 含 $6 \mathrm{~mol} / \mathrm{L} \mathrm{GuHCl}$ 和 $1 \mathrm{mmol} / \mathrm{L}$ EDTA 的 $0.1 \mathrm{~mol} / \mathrm{L}$ Tris $-\mathrm{HCl}$ 缓冲液中用 DTNB 测定 放基含量 ${ }^{[9]}$. PDI 䘪基含量非常稳定, 在 $5 \mathrm{~h}$ 以内, 基本不变.

\section{2 疏基修饰}

完全还原的 PDI 与 $0.5-10$ 倍 PDI 璇基的 IAN 在 $\mathrm{pH} 8.0$ 含 $1 \mathrm{mmol} / \mathrm{L}$ EDTA 的 $0.1 \mathrm{~mol} / \mathrm{L}$ Tris $-\mathrm{HCl}$ 的缓冲液中, 在室温反应 $10-120 \mathrm{~min}$. 过量的修饰剂用 Sephadex G25 离 心凝胶过滤除去, 疏基修饰程度用 DTNB 测定剩余疏基 ${ }^{[9]}$ 来估价.

PDI 活力测定用 sRNase 作底物测定 PDI 的活力 ${ }^{[6]}$. 当PDI 用 GSH/GSSG 氧化还原缓 冲液处理时, 用相同浓度的 GSH/GSSG 代替反应缓冲液中的 DTT.

\section{2 实 验 结 果}

\section{1 必需永基的数目}

新鲜提取的 PDI 在 $6 \mathrm{~mol} / \mathrm{L} \mathrm{GuHCl}$ 中完全变性后, 用 DTNB 测到每个亚基有 2.7 个疏 基, 用 DTT 完全还原 PDI 含 6.6 个疏基. 表明纯化的酶每个亚基含 3 个疏基和两对二硫键.

PDI 在没有被 DTT 还原前不表现异构酶活力, 也不能被 IAN 修饰. 完全还原的酶被 IAN 修饰时, 活力下降, 和 IAA 修饰 ${ }^{[4]}$ 一样最多只有 2 个疏基能被 IAN 修饰. 图 1(a) 是用 邹氏作图法 ${ }^{[10]}$ 得到的修饰程度与剩余活力的数量关系. 结果表明 2 个被修饰的䟽基都是必 需的,修饰其中 1 个就会导致整个酶分子失活. 这个结果与 Hawkins ${ }^{[4]}$ 和 Vuori ${ }^{\left[{ }^{[5]}\right.}$ 的结果不

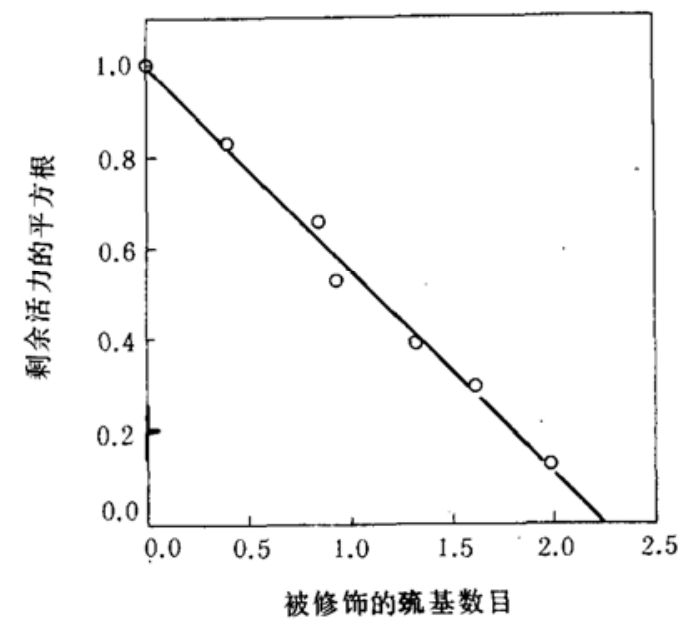

(a)

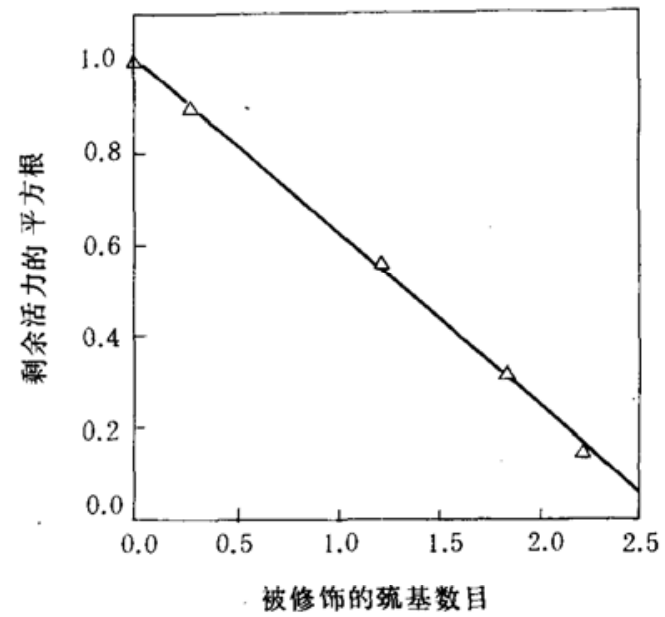

(b)

图 1 IAN 和 IAA 修饰流基对 PDI 活力的影响 ${ }^{[10]}$

以剩余活力的平方根 $A^{1 / 2}$ 对被修饰毓基的数目作图. 䀲在 $0.1 \mathrm{~mol} / \mathrm{L}$ Tris-HCl, $1 \mathrm{mmol} / \mathrm{L}$ EDTA, pH8.0 的缓冲液中, 用不同量的 IAN(a) 修饰; (b) 是对文献[4] 的数据用邹氏作图法进行处理 
一致. 当我们用邹氏作图法处理 Hawkins 的数据时,也得到和我们相同的结果 (图 1(b)).

\subsection{PDI 在 GSH/GSSG 無化还原缓冲液中的激活过程}

图 2 描述 PDI 在 GSH/GSSG $=4$ 的氧化还原缓冲液中的激活过程, PDI 在还原的氧化还 原缓冲液中很快被激活，在 $4 \mathrm{~min}$ 后达到最大值.

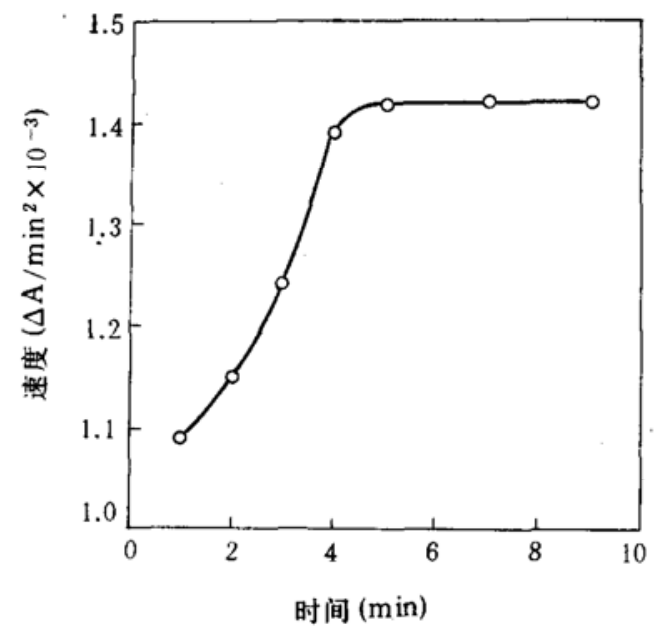

图 2 在氧化还原缓冲液中, PDI 催化 sRNase 形成 RNase 的过程

PDI $1 \mu \mathrm{g} / \mathrm{ml}$ 在 $\mathrm{pH} 7.5$ 含有 $1.2 \mathrm{mmol} / \mathrm{L} \mathrm{GSH}, 0.3 \mathrm{mmol} / \mathrm{L}$ GSSG 和 $2 \mathrm{mmol} / \mathrm{L}$ EDTA 的 $0.2 \mathrm{~mol} / \mathrm{L}$ 磷酸钠缓冲液中于 $30^{\circ} \mathrm{C}$ 保温不同时间, 再加人 sRnase 至 $0.05 \mathrm{mg} / \mathrm{ml}$, 测定 PDI 的活力

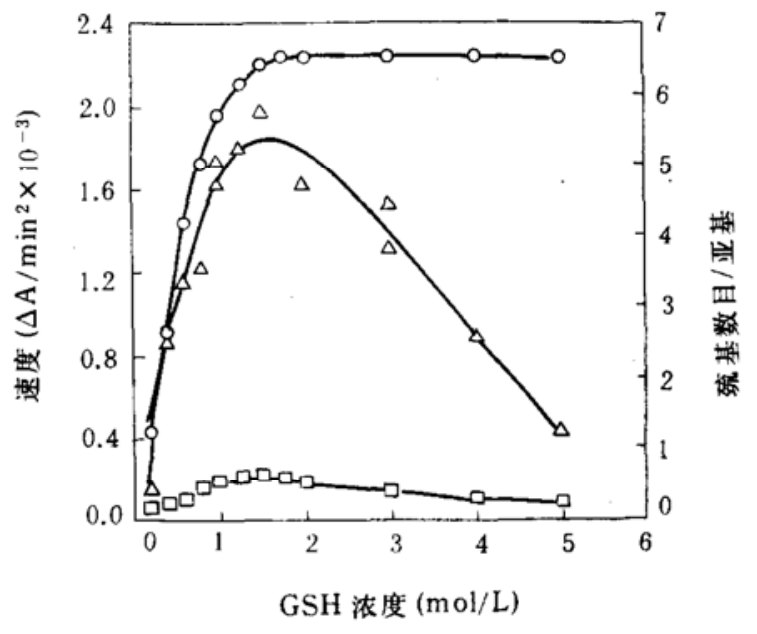

图 3 不同 GSH 浓度对 PDI 的疏基含量和活力 的影响

GSSG 浓度为 $0.3 \mathrm{mmol} / \mathrm{L}$, 不同浓度的 GSH, 于 $\mathrm{pH} 7.5$ 的含 $2 \mathrm{mmol} / \mathrm{L}$ EDTA 的 $0.2 \mathrm{~mol} / \mathrm{L}$ 磷酸钠缓冲液中保温 $10 \mathrm{~min}$. 其他条件同图 2. (O) 䔩基含量, $(\triangle)$ PDI 活力, $(\square)$ 无 PDI 时 sR Nase 的自复活

\subsection{GSH/GSSG 比率对 PDI 旈基含每和活力的影响}

GSH/GSSG 混合物的氧化还原电位决定于 GSH/GSSG 的比率, GSH/GSSG 的比率对 PDI 活力和流基含量的影响见图 3. PDI 的活力和琉基含量随着比率的增加很快上升, 在比 率为 4 时活力和颈基含量都达到最大值, 比率进一步升高时, PDI 的活力明显下降, 但䟽基含 量保持不变.

\section{3 讨 论}

虽然从牛肝 cDNA 所推出的氨基酸序列中, 只有 6 个 Cys 残基 ${ }^{[1]}$, 所有用 DTNB 测定的 结果都表明 PDI 1 个亚基上有 7 个 $\mathrm{Cys}^{[2,3]}$. 用 DTNB 测定旒基时,有可能由于反应不完全 而会使实测数据偏小, 因此如何解释这种差别, 还有待进一步研究. 我们所制备的 PDI 每个 亚基含 3 个自由䟽基和两对二硫键, 这与 Carmichael ${ }^{[2]}$ 的结果一致, 但 Gilbert ${ }^{[3]}$ 和 Hawkins ${ }^{[4]}$ 报道有三对二硫键. PDI 是 1 个巯基二硫键氧化还原酶, 活性部位的氧化还原电位为 -0.11 $\mathrm{V}$, 因此是一个很好的氧化剂, 催化底物分子中二硫键的形成, 而本身的二硫键很容易被还原 ${ }^{[4]}$. 在 PDI 制备和保存过程中, 由于篻化还原条件的差异, 颈基二硫键的状态有所不同, 是完全有 可能的.

Hawkins $^{[4]}$ 等报道 PDI 被 IAA 修饰的疏基是位于和硫氧还蛋白活性中心类似肽段的 Cys 32 和 Cys 379. 定点突变的结果表明这 2 个必需䘪基是相互独立的, 每个 PDI 亚基含 2 个相 
互独立的活性中心 ${ }^{[5]}$. 本文的结果和 Hawkins ${ }^{[4]}$ 的结果不同, 但用邹氏作图法 ${ }^{[10]}$ 重新处理他 们的数据得到和我们相同的结论, 即修饰这 2 个必需基团中的一个, 就导致酶完全失活, 这与 定点突变的结果不一致. 很多实验数据表明 (文献[11]), 化学修饰和定点突变得出不同的结果 是常见的, 这可能是由于化学修饰剂本身引人的诸如空间位阻等因素而产生的. 尽管定点突 变的结果表明 2 个活性中心是相互独立的, 但当 PDI 的 1 个颈基被 IAN 修饰后, 导致酶分子 完全失活. 我们推测 2 个必需基团虽然处在序列的不同部位, 各自可以独立催化䟽基二硫键 交换反应,但它们在空间位置上离得很近, 当 PDI 的 1 个疏基被 IAA 或 IAN 修饰后, 所引人 的修饰基团, 导致另 1 个疏基不能与底物接触, 从而使酶完全失活. Hawkins ${ }^{[8]}$ 等也曾报道, 2 个必需基团可以用 $\mathrm{N}, \mathrm{N}^{\prime}$-bis(iodoacetamide) 交联在一起, 说明它们在空间位置上的确离得很 近.

Hawkins $^{[4]}$ 等曾提出 PDI 的䟽基被修饰后, 就阻断了活性中心内部疏基/二硫键之间的 转换, 从而使酶失活, 这似乎意味着活性中心可以形成的二硫键是 PDI 活性所必需的. 本文 的结果表明当 PDI 所有的疏基都处于还原状态时, 酶活性达到最大, 所以, PDI 的二硫键不是 异构酶活性所必需的. 当 GSH 浓度继续升高时, 酶活力反而降低可能是产物的天然二硫键 被还原或酶和底物形成的中间物的二硫键被还原而引起. 从结果看来 PDI 催化䟽基二硫键 交换时, 可能并不一定需要活性部位旒基/二硫键的转换.

致谢感谢邹承鲁先生对本工作的精心指导和王志珍老师帮助修改论文.

\section{参 考 文 献}

[1] Parkkonen, T. et al., Biochem. J., 1988, 256:1005-1011.

[2] Carmichael, D. F. et al., J. Biol. Chem., 1979, 254:8386-8390.

[3] Gilbert, H. F., Biochemistry, 1989, 28:7298-7305.

[4] Hawkins, H. C. et al., Biochem. J., 1991, 275:335-339.

[5] Vuori, K. et al., J. Biol. Chem., 1992, 267:7211-7214.

[6] Lambert, N. et al., Biochem. J., 1983, 213:225-234.

[7] Lyles, M. M. et al., Biochemistry, 1991, 30:613-619.

[ 8] Hawkins, H. C. et al., Biochem. J., 1991, 275:341-348.

[9] Ellman, G. L., Arch. Biochem. Biophys., 1959, 82:70-77.

[10] Tsou, C. L., Scientia Sinica, 1962, 6:1535-1558.

[11] Profy, A. T., Schimmel, P. C., Progress in Nucleic Acid Res. and Mol. Biol., 1990, 35:1-26. 